\title{
Fibrosis markers and CRIM1 increase in chronic heart failure of increasing severity
}

\author{
Ermanno Eleuteri ${ }^{1} *^{*}$, Antonino Di Stefano ${ }^{1 * \#}$, Davide Vallese ${ }^{1}$, Isabella Gnemmi ${ }^{1}$, Alessandro Pitruzzella ${ }^{1}$, Franco \\ Tarro Genta ${ }^{2}$, Lorena Delle Donne ${ }^{1}$, Francesco Cappello ${ }^{3,4}$, Fabio L. M. Ricciardolo ${ }^{5}$, and Pantaleo Giannuzzi ${ }^{1}$ \\ ${ }^{1}$ Divisione di Cardiologia Riabilitativa e Laboratorio di Citoimmunopatologia Apparato Cardio-Respiratorio, Fondazione Salvatore Maugeri, IRCCS, \\ Veruno, NO, Italy, ${ }^{2}$ Divisione di Cardiologia Riabilitativa, Clinica Major, Torino, Italy, ${ }^{3}$ Dipartimento di Biomedicina Sperimentale e Neuroscienze \\ Cliniche, Sezione di Anatomia Umana, Università di Palermo, Istituto Euro-Mediterraneo di Scienza e Tecnologia, Palermo, Italy, ${ }^{4}$ Istituto Paolo \\ Sotgiu, Libera Università degli Studi di Scienze Umane e Tecnologiche, Lugano, Switzerland, and ${ }^{5}$ Division of Respiratory Disease, A.O.U. San Luigi \\ Hospital, Department of Clinical and Biological Sciences, University of Torino, Orbassano, Torino, Italy
}

\begin{abstract}
Background: Fibrosis suppressors/activators in chronic heart failure (CHF) is a topic of investigation.

Aim: To quantify serum levels of fibrosis regulators in CHF.

Methods: ELISA tests were used to quantify fibrosis regulators, procollagen type-(PIP)I, (PIP)III, collagen-I, III, BMP1,2,3,7, SDF1 $\alpha$, CXCR4, fibulin 1,2,3, BMPER, CRIM1 and BAMBI in 66 CHF (NYHA class I, $n=9$; II, $n=34$; III $n=23$ ), and in 14 controls.

Results: In CHF, TGF $\beta$ R2, PIPIII, SDF1 $\alpha$ and CRIM1 were increased. PIPIII correlated with CRIM1. Conclusions: The BMPs inhibitor CRIM1 is increased and correlates with higher levels of serum PIPIII showing an imbalance in favor of pro-fibrotic mechanisms in CHF.
\end{abstract}

\author{
Keywords \\ Endothelial dysfunction, heart fibrosis, \\ inflammation
}

\section{History}

Received 28 January 2014

Revised 18 February 2014

Accepted 18 February 2014

Published online 11 March 2014

\section{Introduction}

Increased collagen synthesis and degradation has been found in heart failure (HF) patients (Fassbach \& Schwartzkopff, 2005; Izawa et al., 2005), and myocardial fibrosis is associated to development of HF (Barasch et al., 2009; Querejeta et al., 2004). Samples coming from both postmortem human hearts (Olivetti et al., 1994; Rossi, 1998; Tanaka et al., 1986) and endomyocardial human biopsies have shown that fibrillar collagen deposition is increased in the myocardium of patients with left ventricular hypertrophy (Brilla et al., 2000; Ciulla et al., 1997; McLenachan \& Dargie, 1990; Schwartzkopff et al., 2000; Weber, 2000). Furthermore, fibrosis is increased in more advanced stages of chronic heart failure (CHF) and is associated with a poor prognosis (Debl et al., 2006; Harris et al., 2006; Moon et al., 2003, 2005; Weidemann et al., 2005).

Though serum concentrations of procollagen type I peptide (PIP) and type III (PIPIII) can be considered as useful markers of collagen type I and III synthesis (López et al., 2001), the metabolic pathways involved in fibrosis are more

\footnotetext{
*These authors contributed equally to the current work.

"E-mail: ermanno.eleuteri@fsm.it (E. Eleuteri), antonino.distefano@ fsm.it (A. Di Stefano).

Address for correspondence: Dr. Ermanno Eleuteri, MD, Divisione di Cardiologia Riabilitativa e Laboratorio di Citoimmunopatologia Apparato Cardio-Respiratorio, Fondazione Salvatore Maugeri, IRCCS, via per Revislate 13, 28010 Veruno, NO, Italy. Tel: +39 0322884914. Fax: +39 0322 884966. E-mail: ermanno.eleuteri@fsm.it
}

complex and the spectrum of molecules that may interact to influence collagen turnover in HF of increasing severity is wide. Transforming growth factor (TGF) $\beta 1$ and its receptors R1 and R2 are involved in down-regulation of inflammation (Aukrust et al., 1999; Dixon et al., 2011), vascular morphogenesis regulation and extracellular matrix synthesis (Jaffe et al., 2012). A pro-angiogenic growth activity and increased cell survival stimulation has been reported for stromal cellderived factor- $1 \alpha($ SDF- $1 \alpha)$ and its receptor CXCR4 (Leone et al., 2006; Rose et al., 2008). Fibroblast growth factors (FGF) 1 and 2 (acidic and basic, respectively) are involved in the regulation of cardiac angiogenesis and repair (Zhao et al., 2011) and FGF2 is required for development of cardiac hypertrophy (House et al., 2010). Fibulins are involved in structural support, matrix-cell interactions and elastogenic activity (Yanagisawa \& Davis, 2010). Circulating bone morphogenic proteins (BMP) 1-3 may have a pro-fibrotic effect in the kidneys (Grgurevic et al., 2011). BMP2 can suppress renal interstitial fibrosis and TGF $\beta 1$ can induce cardiac fibrosis (Wang et al., 2012; Yang et al., 2011). BMP4 can reduce TGF $\beta 1$-induced extracellular matrix tenascin $\mathrm{C}$ and fibronectin production in cultured lung fibroblasts (Pegorier et al., 2010). BMP4 inhibitor gremlin is overexpressed in idiopathic pulmonary fibrosis (Myllärniemi et al., 2008). BMP7 suppresses hepatic fibrosis (Yang et al., 2012), limits Smad3 DNA binding and decreases renal fibrosis (Luo et al., 2010), reduces collagen content in asbestos-treated mice (Myllärniemi et al., 2008), and preserves the endothelial cardiac phenotype preventing fibroblast 
formation and fibrosis (Zeisberg et al., 2003). BMP endothelial cell precursor-derived regulator (BMPER) interacts with BMPs and when overexpressed antagonizes their function (Heinke et al., 2008) or regulates BMPs function (Moser et al., 2007). Cysteine-rich motor neuron 1 (CRIM1) interacts with BMPs and acts as an antagonist reducing production and processing of mature BMPs and decreasing secretion of BMPs (Glienke et al., 2002; Wilkinson et al., 2003). The BMP and activin membrane-bound inhibitor (BAMBI) is a membrane-spanning glycoprotein that acts as a negative regulator of TGF $\beta$ signaling (Sekiya et al., 2004). A prevalent anti-fibrotic activity was postulated for the pseudo-receptor BAMBI that results up-regulated by TGF $\beta 1$ stimulation in the endothelium (Xavier et al., 2010).

The aim of the present study was to better describe the equilibrium between levels of serum/plasma biomarkers of a selected series of fibrosis activators, fibrosis suppressors and regulators in patients with CHF of increasing severity, to compare them with values of a healthy control group of subjects, and to evaluate possible correlations between fibrosis regulators and heart echocardiographic and functional indexes.

\section{Methods}

\section{Study population}

The study was designed as a prospective, observational trial. The total study population consisted of 80 subjects: 66 patients with CHF (9 in NYHA class I, 34 in NYHA II and 23 in NYHA III), 14 healthy volunteers matched for age and sex. Selection/exclusion criteria for CHF patients were as follows: (1) history of ischemic or idiopathic dilated cardiomyopathy with symptoms and clinical episodes of HF; (2) sinus rhythm, stable medications and no exacerbation of symptoms during the preceding 6 months; (3) echocardiographic left ventricular ejection fraction (LVEF) $<40 \%$; (4) absence of angina and/or instrumentally inducible myocardial ischemia and/or complex ventricular arrhythmias; (5) absence of severe anemia (hemoglobin $<10 \mathrm{~g} / \mathrm{dl}$ ), renal insufficiency (creatinine $<2 \mathrm{mg} / \mathrm{dl}$ ) or hepatic insufficiency (AST $<50 \mathrm{U} / \mathrm{l}$, ALT $<50 \mathrm{U} / \mathrm{l})$. Clinical information, including demographics, co-morbidities, medications, and symptom level based on NYHA classification were collected before enrolment. In the following week, all patients underwent clinical and echocardiographic evaluation, 6-minute walking test (6'WT), ergospirometric test, and blood sample collection. Characteristics of patients such as mean age, LVEF, sex, performance at 6'WT and ergospirometric test $\left(\mathrm{VO}_{2} \mathrm{max}\right)$, and etiology of cardiac disease (ischemic heart disease, IHD or idiopathic cardiomyopathy, ICM) are summarized in Tables 1 and 2 . Table 2 shows a comparative analysis for age, LVEF\%, 6'WT and $\mathrm{VO}_{2}$ max between the IHD and ICM subgroups.

The study was carried out in conformity with the Declaration of Helsinki. The protocol was approved by the Central Ethics Committee of the Salvatore Maugeri Foundation and informed written consent was obtained from all participants in the study.

\section{Echocardiographic evaluation}

At enrolment, all subjects underwent complete echocardiographic and Doppler evaluation by a single and experienced operator (EE). Left ventricular volumes (LVV) were calculated from orthogonal apical views using the biplane arealength method, and ejection fraction (LVEF\%) was derived from the standard equation. Intra-observer variability for LVV and LVEF\% was, respectively, $6 \pm 2 \mathrm{ml}$ and $2 \pm 1 \%$. Deceleration time (DT) of early filling (ms) was measured, as a strong predictor of pulmonary capillary wedge pressure in patients with left ventricular dysfunction (Giannuzzi et al., 1994).

\section{Ergometric evaluation}

All tests were performed on a cycle ergometer (Ergo-metrics 800S; Sensormedics; Yorba Linda, CA). After a 1-min unloaded-cycling warm-up period, a ramp protocol of $10 \mathrm{~W} / \mathrm{min}$ (CHF-TR and CHF-C) or $15 \mathrm{~W} / \mathrm{min}(\mathrm{N})$ at a pedaling rate of $60 \mathrm{revs} / \mathrm{min}$ was started and participants were encouraged to exercise until exhaustion. Respiratory gas exchange measurements were obtained breath-by-breath using a computerized metabolic cart (Vmax29; Sensormedics; Yorba Linda, CA). Peak oxygen uptake $\left(\mathrm{VO}_{2}\right)$ was the mean $\mathrm{VO}_{2}$ value observed during the last $30 \mathrm{~s}$ of the exercise period. Predicted $\mathrm{VO}_{2 \max }$ was determined using a sex-, age-, and protocol-specific formula defined by Wasserman et al. (2005), and the ventilatory anaerobic threshold (VAT) was estimated by the V-slope and/or respiratory equivalents methods.

Table 2. Characteristics of ischemic and idiopathic CHF patients.

\begin{tabular}{lcccc}
\hline Subjects $(n)$ & Age & LVEF\% & 6 'WT & $\mathrm{VO}_{2}$ max \\
\hline IHD (39) & $65 \pm 2$ & $26 \pm 1$ & $401 \pm 18$ & $15 \pm 1$ \\
ICM (27) & $55 \pm 3^{*}$ & $24 \pm 1$ & $439 \pm 28$ & $20 \pm 2^{* *}$ \\
\hline
\end{tabular}

Data expressed as mean $\pm \mathrm{SE}$; IHD, ischemic heart disease; ICM, idiopathic cardiomyopathy; LVEF, left ventricular ejection fraction; 6WT, 6-minute walking text; $\mathrm{VO}_{2}$ max, maximal oxygen consumption. ANOVA: $* p=0.03 ; * * p=0.010$.

Table 1. Characteristics of CHF patients and healthy controls.

\begin{tabular}{lcclccc}
\hline Subjects $(n)$ & Age & 6WT & LVEF\% & $\mathrm{VO}_{2} \max$ & Sex (M/F) & IHD/ICM \\
\hline Controls (14) & $53 \pm 2$ & - & $60 \pm 2$ & - & $14 / 0$ & - \\
NYHA I (9) & $55 \pm 4$ & $486 \pm 23$ & $25 \pm 2$ & $20.8 \pm 2.3$ & $8 / 0$ & $4 / 5$ \\
NYHA II (34) & $63 \pm 2^{*}$ & $405 \pm 14$ & $27 \pm 1$ & $16 \pm 1$ & $31 / 3$ & $22 / 12$ \\
NYHA III (23) & $65 \pm 2^{*}$ & $276 \pm 31$ & $23 \pm 1^{* *}$ & $13 \pm 1$ & $16 / 7$ & $13 / 10$ \\
\hline
\end{tabular}

Data expressed as mean \pm SE; IHD, ischemic heart disease; ICM, idiopathic cardiomyopathy; LVEF, left ventricular ejection fraction; 6WT', 6-minute walking test; $\mathrm{VO}_{2} \mathrm{max}$, maximal oxygen consumption.

ANOVA: $* p<0.05$ from NYHA I; $* * p<0.05$ from NYHA II. 


\section{Serum collection and analysis}

Blood samples were collected using Bekton Dikinson (BD) Vacutainer Cat Plus REF 367896 for serum. Serum or plasma was then aliquoted and immediately frozen at $-80^{\circ} \mathrm{C}$ until analysis. The serum/plasma levels of all molecules (see Table 3) were determined by commercially available enzyme linked immunosorbent assay (ELISA) kits as described in Table 3. Table 3 also shows the lower detection limits and the method of quantification (ELISA) for each of the molecules studied. Manufacturers' instructions were carefully followed for each of the ELISA kits used. Further details for each kit are available from the respective online datasheet.

\section{Data analysis}

Group data were expressed as mean \pm standard error for functional data or median (range or interquartile range, IQR) for serum/plasma levels. Differences between groups were analysed using analysis of variance (ANOVA) for functional data. The Kruskal-Wallis test was applied for serum/plasma data followed, when differences were significant, by the Mann-Whitney U test for comparison between groups. Single regression analysis was performed using the Spearman correlation test. Probability values of $p<0.05$ were considered as significant. Data analysis was performed using the Stat View SE Graphics program (Abacus Concepts Inc., Berkeley, CA).

\section{Results}

\section{Subjects}

Characteristics of the study population are summarized in Table 1. Patients in NYHA class II and III were slightly older than NYHA I patients. IHD patients were slightly older $(p=0.03)$ compared to ICM subjects and showed a significantly lower $\mathrm{VO}_{2} \max (p=0.01)$ (see Table 2 for characteristics of patients when divided into ischemic and idiopathic subgroups).

\section{Quantification of serum/plasma markers}

Quantification of FGF basic and acidic and of TGF $\beta 1$ did not differ significantly in the four groups, showing a low presence of these molecules both in patients and controls (Table 4). TGF $\beta$ R 1 was slightly decreased in NYHA class II (0.036) and III $(p=0.020)$ compared to controls, even though this difference was not apparent on the Kruskal-Wallis test for multiple comparisons. TGF $\beta$ R2 was significantly higher in NYHA class III compared to NYHA class II $(p=0.011)$ and controls $(p=0.018)$ (Figure 1a). The TGF $\beta$ pseudo-receptor BAMBI was similarly expressed in all groups, showing a low expression in the four groups examined. The pro-angiogenic factor SDF1 $\alpha$ was significantly increased in NYHA class III compared to NYHA class II $(p=0.013)$, NYHA class I $(p=0.014)$ and controls $(p=0.0006)$ (Figure $1 b)$, at variance with its receptor CXCR4, which was similarly expressed in the four groups. Fibulins 1 and 2 were highly expressed in all subjects without any significant differences between groups. Fibulin 4 was significantly higher in NYHA class III patients than NYHA I $(p=0.011)$, even though this difference was not confirmed by the more restrictive statistical analysis for multiple comparisons (Kruskal-Wallis test). Among the BMP proteins analysed (BMP1, BMP2, BMP3, BMP7, BMP10), BMP1 and BMP2 were the most abundantly expressed in all subjects without significant differences between groups. BMPER, as well, was modestly expressed, at concentrations similar to BMP2, in all subjects without significant differences between groups. Interestingly, the BMP antagonist CRIM1 was significantly higher in NYHA class III patients

Table 3. Summary of the ELISA assays performed in the serum/plasma for biomarkers included in the study.

\begin{tabular}{|c|c|c|c|c|}
\hline Molecules & Manufacturer (code) & Lower detection limit & Type of plasma/serum & Analytical method \\
\hline FGF acidic & R\&D Systems DFA00B & $5.68 \mathrm{pg} / \mathrm{ml}$ & Serum & ELISA \\
\hline TGF- $\beta$ R1 & USC Life Science Inc. E90397Hu & $0.118 \mathrm{ng} / \mathrm{ml}$ & Serum & ELISA \\
\hline TGF- $\beta$ R2 & USC Life Science Inc. E92972Hu & $0.126 \mathrm{ng} / \mathrm{ml}$ & Serum & ELISA \\
\hline BAMBI & USC Life Science Inc. E98566Hu & $0.064 \mathrm{ng} / \mathrm{ml}$ & Serum & ELISA \\
\hline SDF- $1 \alpha$ & $\begin{array}{l}\text { R\&D Systems } \\
\text { DSA00 }\end{array}$ & $18 \mathrm{pg} / \mathrm{ml}$ & Plasma & ELISA \\
\hline Fibulin 1 & USC Life Science Inc. E92472Hu & $0.23 \mathrm{ng} / \mathrm{ml}$ & Serum & ELISA \\
\hline Fibulin 2 & USC Life Science Inc. E93152Hu & $5.6 \mathrm{pg} / \mathrm{ml}$ & Serum & ELISA \\
\hline Fibulin 4 & USC Life Science Inc. E95421Hu & $1.12 \mathrm{ng} / \mathrm{ml}$ & Serum & ELISA \\
\hline BMP-1 & USC Life Science Inc. E90653Hu & $0.064 \mathrm{ng} / \mathrm{ml}$ & Serum & ELISA \\
\hline BMP-2 & USC Life Science Inc. E90013Hu & $15.98 \mathrm{pg} / \mathrm{ml}$ & Serum & ELISA \\
\hline PIP-I & TaKaRa MK101 & $10 \mathrm{ng} / \mathrm{ml}$ & Serum & ELISA \\
\hline PIPIII & USC Life Science Inc. E90573Hu & $12.8 \mathrm{pg} / \mathrm{ml}$ & Serum & ELISA \\
\hline Collagen I & USC Life Science Inc. E90571Hu & $0.237 \mathrm{ng} / \mathrm{ml}$ & Serum & ELISA \\
\hline Collagen III & USC Life Science Inc. E90176Hu & $13.1 \mathrm{pg} / \mathrm{ml}$ & Serum & ELISA \\
\hline
\end{tabular}


Table 4. Quantification of pro-fibrotic molecules and growth factors in the serum/plasma of patients with chronic heart failure and control subjects.

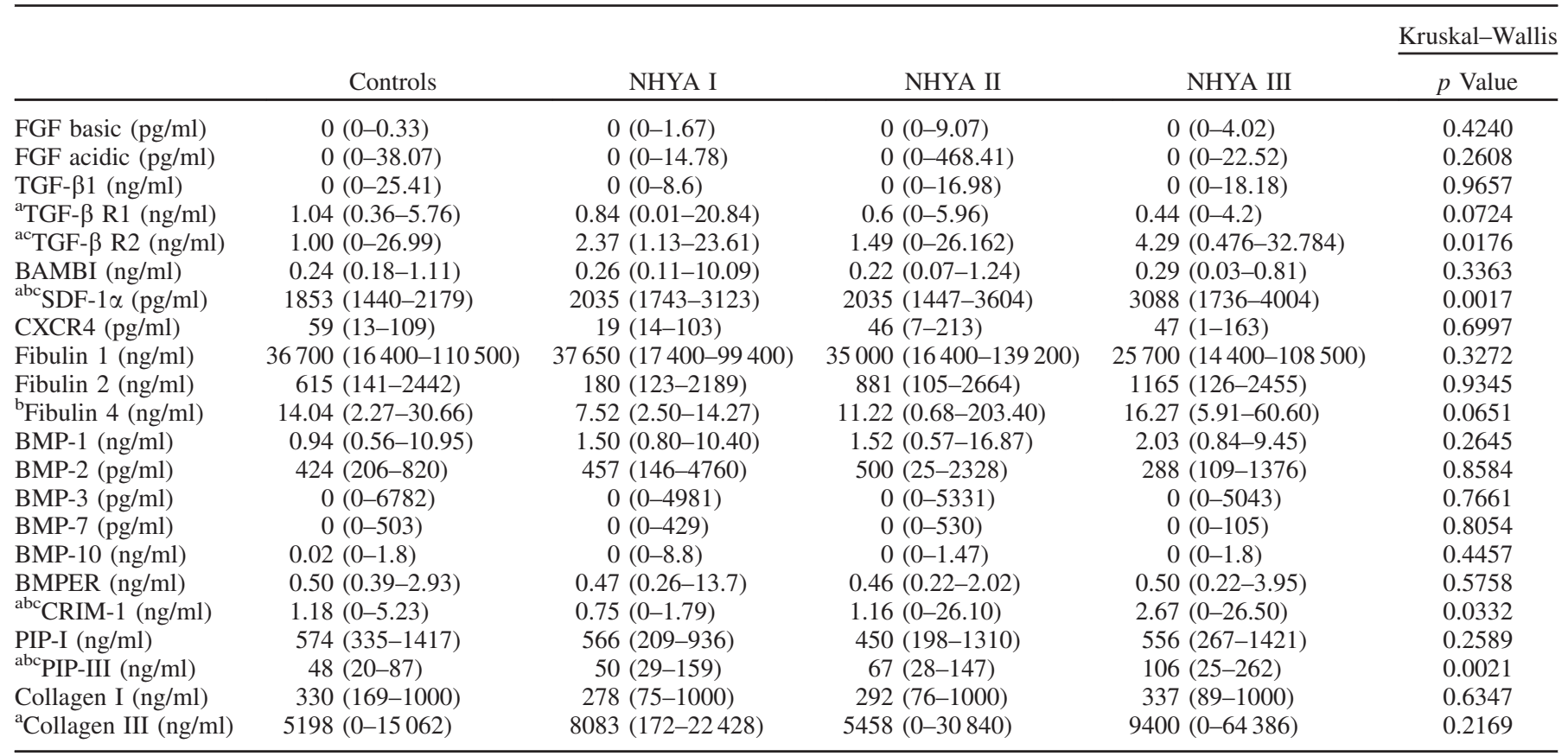

Data expressed as median (range).

Mann-Whitney U test: ${ }^{a}$ significantly different from controls $p<0.05 ;{ }^{\mathrm{b}}$ significantly different from NHYA I $p<0.05$; ${ }^{\mathrm{c}}$ significantly different from NHYA II $p<0.05$.
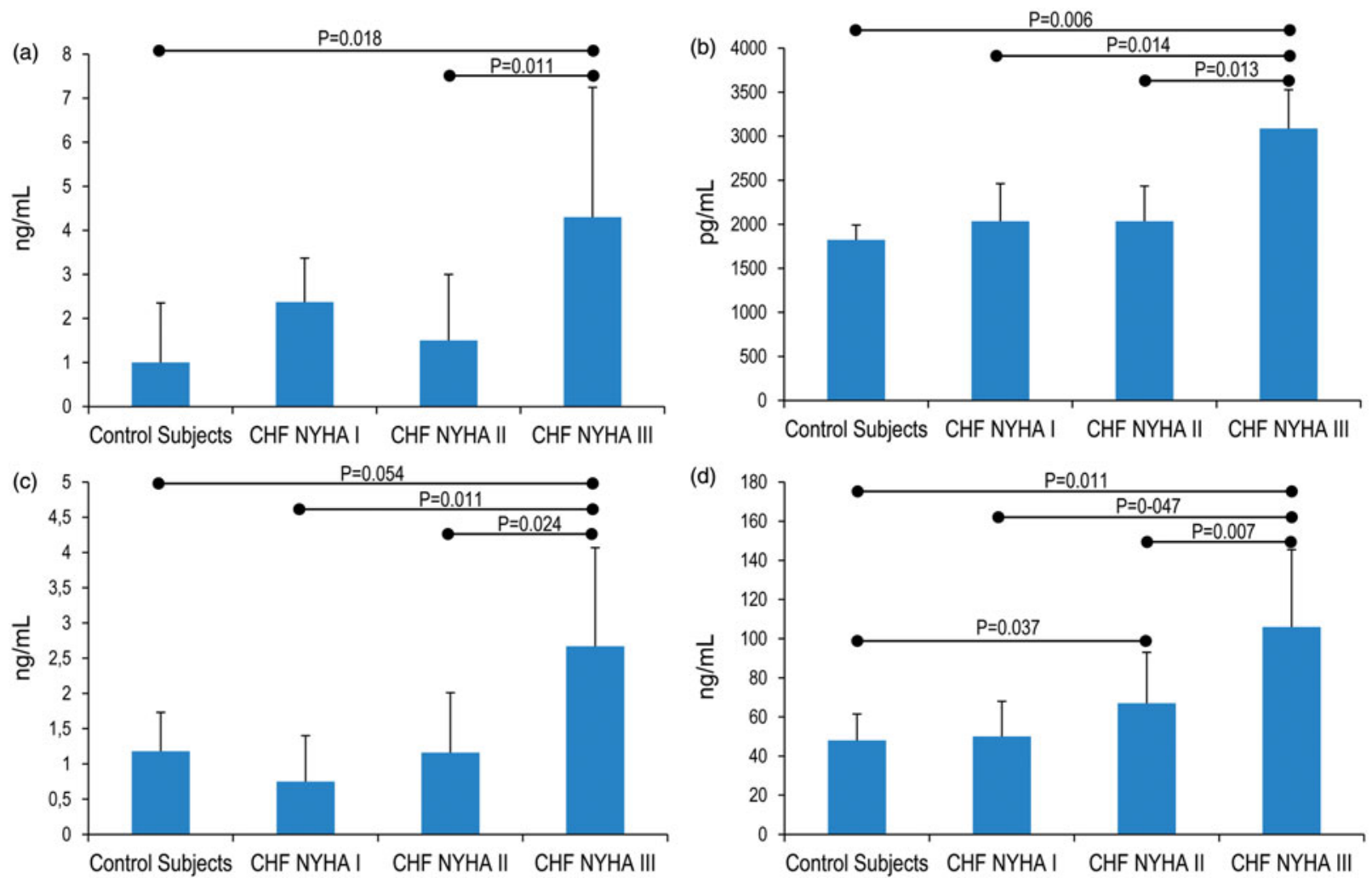

Figure 1. Transforming growth factor- $\beta$ receptor 2 (TGF $\beta$ R2) (a), stromal cell-derived factor-1 $\alpha$ (SDF1 $\alpha$ ) (b), cysteine-rich motor neuron-1 (CRIM1) (c), pro-collagen type III (PIPIII) (d), in serum/plasma of subjects with chronic heart failure from NYHA class I, II, III and healthy controls. The results are expressed as median (interquartile (IQR) range). Statistical analysis: Kruskal-Wallis followed by Mann Whitney U test for comparison between groups. Control subjects: $n=14$; CHF NYHA class I: $n=9$; CHF NYHA class II: $n=34$; CHF NYHA class III: $n=23$.

compared to NYHA class II $(p=0.024)$, NYHA class I $(p=0.011)$ and controls $(p=0.054)$ (Figure 1c). Collagen I and PIP were similarly expressed in the four groups of patients studied. Collagen III was significantly higher in
NYHA class III patients than controls $(p=0.043)$. Most importantly, PIPIII was significantly higher in NYHA class III patients compared to NYHA class II $(p=0.0078)$, NYHA class I $(p=0.047)$ and controls $(p=0.0011)$ (Figure 1d). 
NYHA class II patients also differed significantly from controls $(p=0.038)$. These results are summarized in Table 4.

When ischemic (IHD) were compared to idiopathic (ICM) patients, only CRIM1 and Fibulin 4 serum concentrations were found significantly higher in IHD compared to ICM patients. These results are summarized in Table 5.

When NYHA class I and II were grouped together and compared with NYHA class III patients, TGF $\beta$ R2 (NYHA class I + II versus III: $1.64(0-26)$ versus $4.29(0.4-32) \mathrm{ng} / \mathrm{ml}$, $p=0.011)$, CRIM1 (1.13(0-26) versus 2.67(0-27) ng/ml, $p=0.008)$, PIPIII (63.7(27-159) versus 105.9(25-262) ng/ml, $p=0.004)$, SDF1 $\alpha$ (2035(1446-3604) versus 3087(17354004) $\mathrm{pg} / \mathrm{ml}, \quad p=0.0019)$ serum levels were markedly increased in NYHA class III.

When we compared CHF patients with $\mathrm{VO}_{2} \leq 16$ and $\mathrm{CHF}$ patients with higher $\mathrm{VO}_{2}$ values, only CRIM1 $\left(\mathrm{VO}_{2}>16\right.$ versus $\mathrm{VO}_{2} \leq 16$ : $0.73(0-2.3)$ versus $2.19(0.48-5.59) \mathrm{ng} / \mathrm{ml}$, $p=0.0009)$ serum levels differed significantly. No significant changes were observed for TGF $\beta$ R2 $(p=0.132)$, PIPIII $(p=0.285)$ and $\operatorname{SDF} 1 \alpha(p=0.157)$ between the two subgroups of $\mathrm{CHF}$ patients divided for $\mathrm{VO}_{2}$ consumption.

\section{Correlations}

In our patient cohort, none of the biomarkers studied correlated significantly with LVEF\% at echocardiography. PIPIII was inversely correlated with 6'WT $(r=-0.37$, $p=0.0086)$ (Figure 2a) and DT $(\mathrm{ms})(r=-0.32, p=0.035)$ (Figure 2c). CRIM1 was inversely correlated with 6'WT $(r=-0.36, p=0.0158)$ (Figure $2 \mathrm{~b})$, peak $\mathrm{VO}_{2}(r=-0.53$, $p=0.0015)$ and DT $(\mathrm{ms})(r=-0.43, p=0.010)$ (Figure $2 \mathrm{~d})$. TGF $\beta$ R2 was inversely correlated with DT (ms) $(r=-0.35$, $p=0.027)$; SDF1 $\alpha$ correlated inversely with peak $\mathrm{VO}_{2}$ $(r=-0.42, p=0.032)$. No other correlations were found between serum/plasma biomarkers and age of patients or physiological/functional parameters. PIPIII positively correlated with TGF $\beta$ 2 $(r=0.29, p=0.026)$ (Figure 3a), CRIM1 $(r=0.38, \quad p=0.005) \quad$ (Figure 3b), Fibulin $4 \quad(r=0.35$,

Table 5. Median values of biomarkers in the serum/plasma of chronic heart failure patients according to ischemic or idiopathic etiology of the disease.

\begin{tabular}{lcccc}
\hline CHF etiology & CRIM 1 $(\mathrm{ng} / \mathrm{ml})$ & Fibulin 4 (ng/ml) & PIPIII (ng/ml) & Collagen III (ng/ml) \\
\hline IHD (39) & $2.2(0-26.49)$ & $15(1.3-60)$ & $83356(26566-246472)$ & $8168(172-64386)$ \\
ICM (27) & $0.74(0-2.17)$ & $8.1(0.68-16.62)$ & $53302(27600-172952)$ & $8261(0-31950)$ \\
$p$-value & 0.0102 & 0.0106 & 0.115 & 0.463 \\
\hline
\end{tabular}

Values are expressed as median (range). ICM, idiopathic cardiomyopathy; IHD, ischemic heart disease; CRIM1, Cysteinerich motor neuron-1; PIPIII, Pro-collagen type III peptide. Mann-Whitney U-test for comparison between groups.
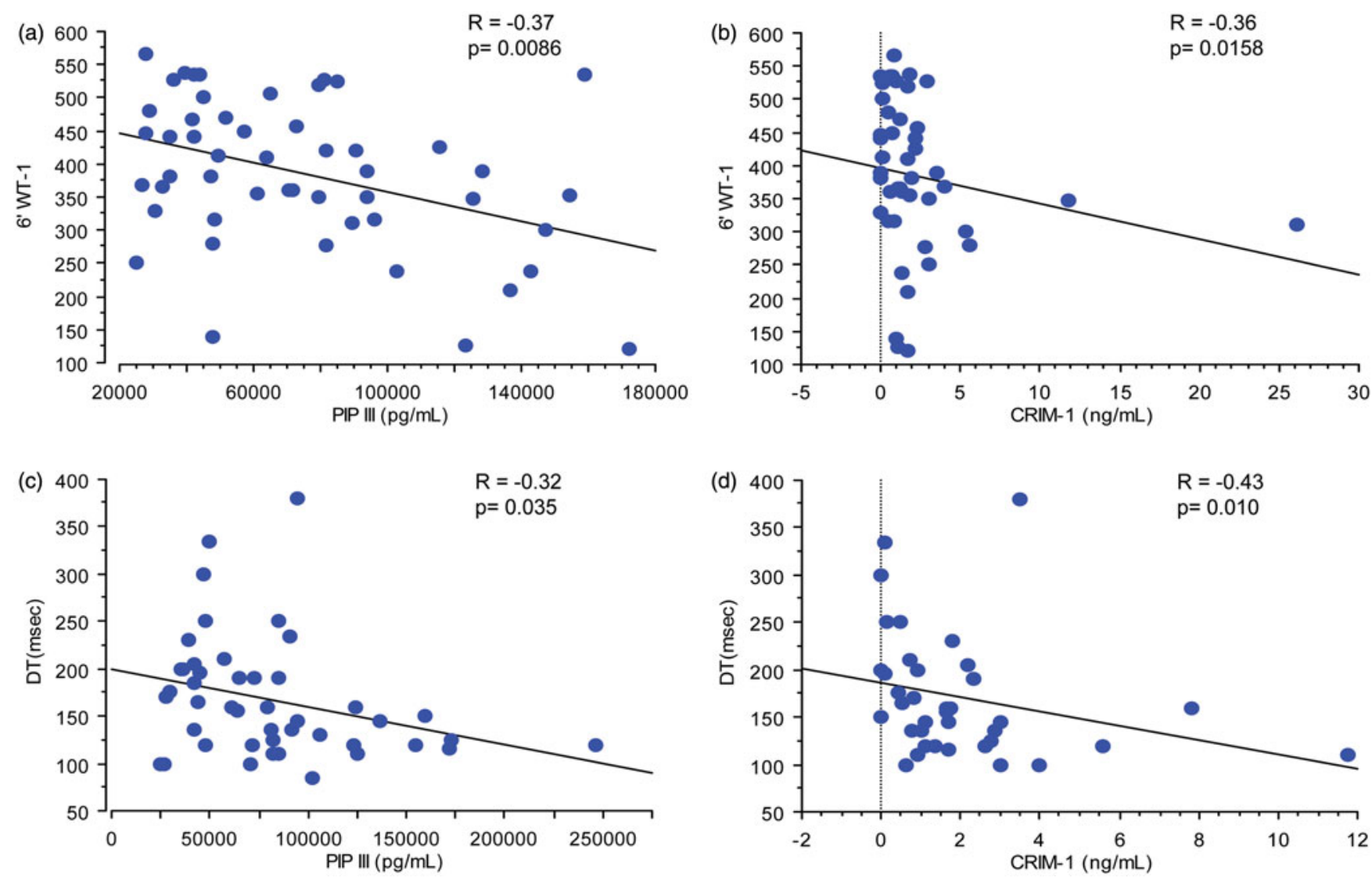

Figure 2. Regression analysis between PIPIII and CRIM1 serum levels and 6-minute walking test (6'WT) (a and b, respectively) and deceleration time (DT-ms) (c and d, respectively) in all patients with CHF $(n=66)$. Spearman's rank correlations. 

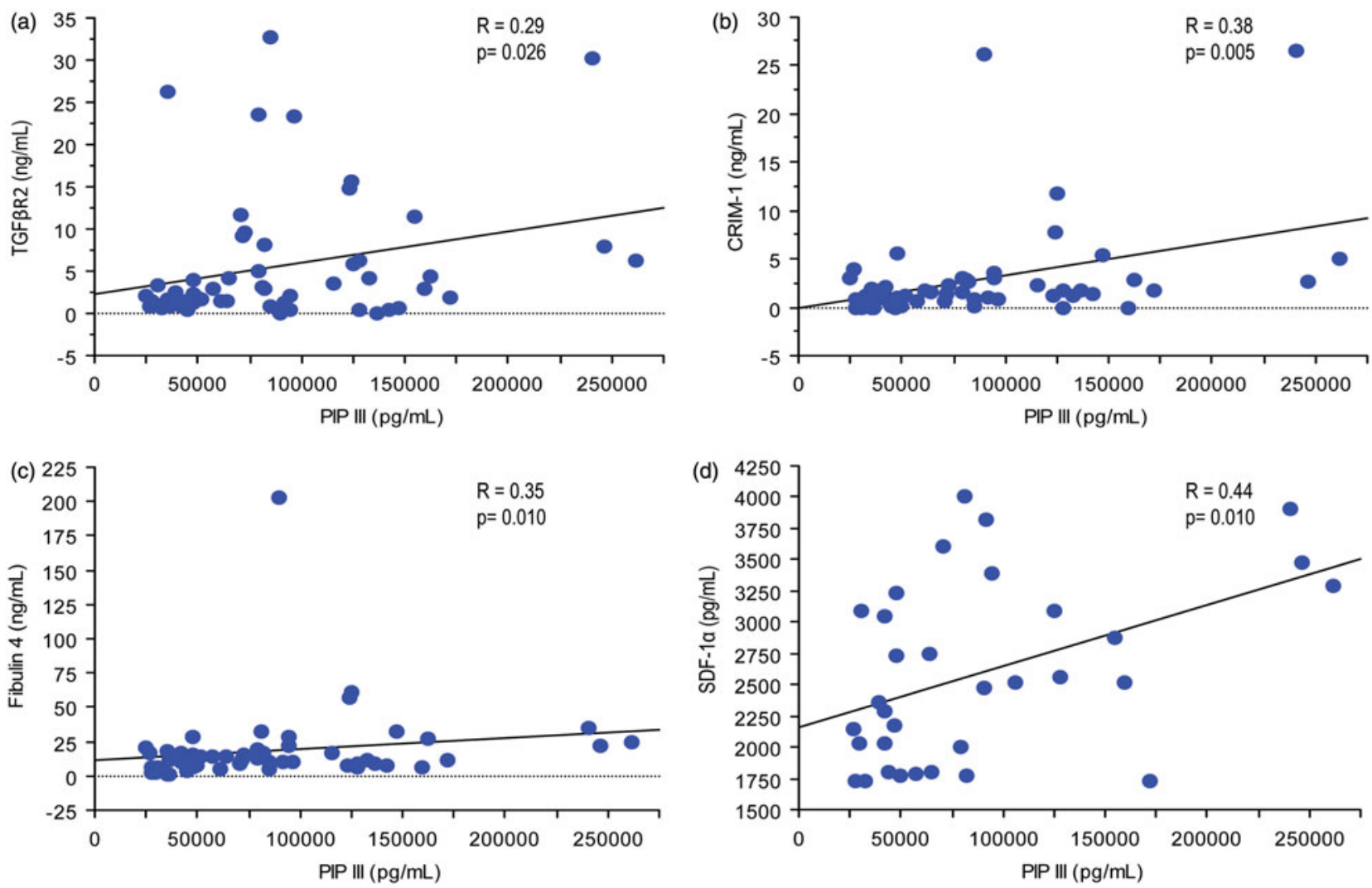

Figure 3. Regression analysis between PIPIII serum levels and pro-fibrotic stimuli TGF $\beta$ R2 (a), CRIM1 (b), fibulin 4 (c) and PIPIII versus a pro-angiogenic stimulator SDF1 $\alpha(\mathrm{d})$ in all patients with CHF $(n=66)$. Spearman's rank correlations.

$p=0.010) \quad($ Figure 3c) and $\operatorname{SDF} 1 \alpha \quad(r=0.44, p=0.013)$ (Figure 3d).

\section{Discussion and conclusions}

In our CHF patients, we demonstrated increasing levels of procollagen type III (PIPIII), TGF $\beta$ R2, stromal cell-derived factor (SDF)-1 $\alpha$ and cysteine-rich motor neuron (CRIM)-1, particularly in patients at a more advanced stage of cardiac disease. PIPIII serum levels correlated inversely with patients' tolerance to dynamic exercise (6'WT) and to the deceleration time of left ventricular early filling (DT), as an echocardiographic index of pulmonary wedge pressure (Giannuzzi et al., 1994). PIPIII serum levels also positively correlated with CRIM-1, SDF-1 $\alpha$, fibulin-4 and TGF $\beta$ R2 in the serum of $\mathrm{CHF}$ patients of increasing severity. These data, taken together, show a progressive imbalance in favor of profibrotic mechanisms with progression of $\mathrm{CHF}$, which can be revealed in the serum/plasma of patients with $\mathrm{CHF}$ of increasing severity.

The role of BMP proteins as anti-fibrotic agents is sufficiently well documented in kidney (Farris \& Colvin, 2012) and liver (Yang et al., 2012), but little is known about their potential involvement in the mechanism of heart fibrosis. Recently it was shown that, in mice, pressure overload-induced collagen deposition was decreased and cardiac function improved after 2 weeks treatment with recombinant BMP-2 (Wang et al., 2012), suggesting a fibrosis antagonizing action for this molecule. In the serum of our CHF patients BMP-1,-2, $-3,-7$ and -10 were poorly represented, BMP-1 and BMP-2 being the most expressed
BMP proteins. None of the BMPs showed significant changes in $\mathrm{CHF}$ patients compared to controls, suggesting that their potential role as anti-fibrotic molecules cannot be exerted in $\mathrm{CHF}$ patients as severity increases. Since a number of molecules, such as BMP endothelial cell precursor derived regulator (BMPER) and cysteine-rich motor neuron 1 (CRIM1), may have a regulatory or inhibitory effect on BMP proteins, we quantified these molecules in the serum of our CHF patients and controls. The BMPER serum levels were relatively low and similar in the four groups of patients studied, suggesting a minor role for this regulatory molecule. Interestingly, and as an original finding, we detected significantly increased serum levels of CRIM-1 in NYHA class III $\mathrm{CHF}$ patients when compared to all the other groups studied; moreover, CRIM-1 serum levels were positively correlated with PIPIII serum levels in CHF patients (Figure 3). A negative significant correlation was also observed between CRIM-1 serum levels and 6'WT and DT(ms), showing, as an original contribution of the present study, a possible relationship between CRIM-1 levels and increased heart fibrosis process, associated to a worse hemodynamic condition (maybe a consequence of diminished left ventricular compliance as reflected by shorter DT) and dynamic exercise performance in the CHF group (Figure 2). In in vitro experiments, CRIM-1 binding of BMP4 and BMP7 leads to a reduction in the production and secretion of mature BMPs (Wilkinson et al., 2003) on metanephric explant cultures. These data, together with our in vivo findings of an increased serum level of CRIM-1, associated to low serum levels of BMPs, prompt speculation on the possible BMPs' inhibitory role of CRIM-1 and subsequent increased heart fibrosis, 
in our patients with CHF of increasing severity. Interestingly, in the sub-group of CHF patients with IHD, CRIM-1 and also fibulin-4, a molecule involved in the elastic fiber assembly, are significantly increased compared to the idiopathic (ICM) group. Also PIPIII levels tended to be higher $(p=0.11)$ in IHD compared to ICM patients (see Table 5), suggesting again a pro-fibrotic role for these proteins in the more compromised patients with CHF. Furthermore, differences for TGF $\beta$ R2, CRIM1, PIPIII and SDF1 $\alpha$ serum levels were markedly significant when NYHA class I and II CHF were grouped and compared with NYHA class III CHF patients, suggesting that the more relevant pro-fibrotic changes are present in the more severe diseased patients. Interestingly, CRIM1 serum levels were significantly higher in CHF patients with $\mathrm{VO}_{2} \leq 16$, when compared with CHF patients with higher $\mathrm{VO}_{2}$ consumption, suggesting again a physiological role for this molecule, particularly on hemodynamic and fibrotic events.

The TGF $\beta$ pseudo-receptor BAMBI, for which a prevalent anti-fibrotic activity has been reported (Onichtchouk et al., 1999; Sekiya et al., 2004), was similarly expressed in the four groups of subjects studied, suggesting a minor role for this protein in controlling the fibrotic changes developing as CHF severity increases.

A pro-angiogenic growth activity has been reported for SDF1 $\alpha$ and its receptor CXCR4 (Leone et al., 2006; Rose et al., 2008). SDF1 $\alpha$ was reported as increased in acute myocardial infarction (AMI) (Leone et al., 2006), suggesting a contribution of bone marrow cells in myocardial regeneration (Leone et al., 2006). In CHF patients, a 3-month physical training program significantly increased serum levels of the pro-angiogenic markers angiopoietin-2 and CD34+ cells, but not of $\mathrm{SDF} 1 \alpha$, as compared with non-trained patients (Eleuteri et al., 2013). In contrast, a down-regulation of pro-angiogenic mechanisms, including SDF1 $\alpha$ reduction, was reported in idiopathic pulmonary fibrosis (Antoniou et al., 2010), suggesting that potentially different mechanisms could be involved in cardiac and lung fibrosis. We here report a significantly increased serum level of SDF1 $\alpha$ in NYHA class III patients compared to all the other groups of subjects and a significant positive correlation of SDF1 $\alpha$ with PIPIII serum levels in patients with CHF of increasing severity (Figures 1 and 3), suggesting a role for this pro-angiogenic molecule in heart remodeling. Since a parallel increase of its receptor CXCR4 was not observed in the plasma of our patients, the effective SDF1 $\alpha$ biological activity developing in these patients needs to be more extensively elucidated by specifically designed in vitro studies. In addition, a potential role of CRIM1 in the process of angiogenesis has also been described (Glienke et al., 2002). CRIM1 is involved in endothelial cell capillary formation in vitro and is expressed in blood vessels in vivo (Glienke et al., 2002). CRIM-1 is strongly upregulated in endothelial cells during tube formation, and is expressed by EC of the inner lining of blood vessels in vivo, suggesting a possible role of CRIM1 in capillary formation and maintenance during angiogenesis. These observations suggest that CRIM1 could be a multifactorial transmembrane protein, involved both in the complex and still only partially known processes of myocardial fibrosis and angiogenesis.
Fibulins have a role in the assembly and stabilization of supramolecular extracellular matrix (ECM) complexes. They also can function as a modulators of cell growth, differentiation and angiogenesis (de Vega et al., 2009). Fibulin-1 and -2 interact with a wide number of ECM complexes; fibulin-4 interacts mainly with tropoelastin (de Vega et al., 2009). Fibulins-1 and -2 were the most expressed fibulins in the serum of our patients without significant differences between groups (Table 4). Fibulin-4 was slightly increased in NYHA class III compared to NYHA class I patients, and it was correlated positively with PIIIP serum levels (Figure 3), probably as a consequence of the more direct relationship of this fibulin type with the cardiac function (de Vega et al., 2009).

Molecules classically considered as fibrosis inductors, such as basic and acidic fibroblast growth factors and TGF $\beta 1$ were poorly recovered in the serum of our patients and controls (Table 4), showing no significant differences between groups. TGF $\beta$ RI was slightly decreased in NYHA class II and III CHF patients, compared to controls. Interestingly, TGF $\beta$ RII serum levels, increased significantly in NYHA class III compared to NYHA class II patients and controls (Table 4) and correlated significantly with PIPIII serum levels (Figure 3), showing a potential role in inducing heart remodelling and fibrosis. Pro-collagen-type I (PIP) and collagen type I were similarly recovered in the serum of the four groups studied. As expected, PIPIII serum level was significantly increased in NYHA class III compared to all the other groups and in NYHA class II compared to controls (Table 4). Serum level of collagen III was also slightly increased in NYHA class III compared to controls (Table 4), confirming the increased fibrotic state and collagen turnover in patients with CHF of increasing severity.

NYHA class II and III patients were slightly older than controls. It can be argued that age may influences serum/plasma biomarker levels. We did not find any significant correlation between age and CRIM1, TGFßRII or SDF1 $\alpha$ serum/plasma levels in all subjects and in patients with $\mathrm{CHF}$ alone, suggesting that age difference between groups likely does not play a significant role in our quantifications.

The prognostic value of the CRIM1 serum test as well as inhibition of the CRIM1 biological functions and upregulation of anti-fibrotic BMP molecules need further specifically planned in vitro and in vivo studies.

\section{Acknowledgements}

The authors thank Ada Patriarca for technical assistance.

\section{Declaration of interest}

This study was supported by Fondazione S. Maugeri, IRCCS, Ricerca Corrente. The authors report no declarations of interest.

\section{References}

Antoniou KM, Soufla G, Lymbouridou R, et al. (2010). Expression analysis of angiogenic growth factors and biological axis CXCL12/ CXCR4 axis in idiopathic pulmonary fibrosis. Connect Tissue Res 51: $71-80$. 
Aukrust P, Ueland T, Lien E, et al. (1999). Cytokine network in congestive heart failure secondary to ischemic or idiopathic dilated cardiomyopathy. Am J Cardiol 83:376-82.

Barasch E, Gottdiener JS, Aurigemma G, et al. (2009). Association between elevated fibrosis markers and heart failure in the elderly: the cardiovascular health study. Circ Heart Fail 2:303-10.

Brilla CG, Funck RC, Rupp H. (2000). Lisinopril-mediated regression of myocardial fibrosis in patients with hypertensive heart disease. Circulation 102:1388-93.

Ciulla M, Paliotti R, Hess DB, et al. (1997). Echocardiographic patterns of myocardial fibrosis in hypertensive patients: endomyocardial biopsy versus ultrasonic tissue characterization. J Am Soc Echocardiogr 10:657-64.

Debl K, Djavidani B, Buchner S, et al. (2006). Delayed hyperenhancement in magnetic resonance imaging of left ventricular hypertrophy caused by aortic stenosis and hypertrophic cardiomyopathy: visualisation of focal fibrosis. Heart 92:1447-51.

de Vega S, Iwamoto T, Yamada Y. (2009). Fibulins: multiple roles in matrix structures and tissue functions. Cell Mol Life Sci 66:1890-902.

Dixon DL, Griggs KM, Bersten AD, De Pasquale CG. (2011). Systemic inflammation and cell activation reflects morbidity in chronic heart failure. Cytokine 56:593-9.

Eleuteri E, Mezzani A, Di Stefano A, et al. (2013). Aerobic training and angiogenesis activation in patients with stable chronic heart failure: a preliminary report. Biomarkers 18:418-24.

Farris AB, Colvin RB. (2012). Renal interstitial fibrosis: mechanisms and evaluation. Curr Opin Nephrol Hypertens 21:289-300.

Fassbach M, Schwartzkopff B. (2005). Elevated serum markers for collagen synthesis in patients with hypertrophic cardiomyopathy and diastolic dysfunction. Z Kardiol 94:328-35.

Giannuzzi P, Imparato A, Temporelli PL, et al. (1994). Doppler-derived mitral deceleration time of early filling as a strong predictor of pulmonary capillary wedge pressure in postinfarction patients with left ventricular systolic dysfunction. J Am Coll Cardiol 23:1630-7.

Glienke J, Sturz A, Menrad A, Thierauch KH. (2002). CRIM1 is involved in endothelial cell capillary formation in vitro and is expressed in blood vessels in vivo. Mech Dev 119:165-75.

Grgurevic L, Macek B, Healy DR, et al. (2011). Circulating bone morphogenetic protein 1-3 isoform increases renal fibrosis. J Am Soc Nephrol 22:681-92.

Harris KM, Spirito P, Maron MS, et al. (2006). Prevalence, clinical profile, and significance of left ventricular remodeling in the endstage phase of hypertrophic cardiomyopathy. Circulation 114:216-25.

Heinke J, Wehofsits L, Zhou Q, et al. (2008). BMPER is an endothelial cell regulator and controls bone morphogenetic protein-4-dependent angiogenesis. Circ Res 103:804-12.

House SL, House BE, Glascock B, et al. (2010). Fibroblast growth factor 2 mediates isoproterenol-induced cardiac hypertrophy through activation of the extracellular regulated kinase. Mol Cell Pharmacol 2: $143-54$.

Izawa H, Murohara T, Nagata K, et al. (2005). Mineralocorticoid receptor antagonism ameliorates left ventricular diastolic dysfunction and myocardial fibrosis in mildly symptomatic patients with idiopathic dilated cardiomyopathy: a pilot study. Circulation 112:2940-5.

Jaffe M, Sesti C, Washington IM, et al. (2012). Transforming growth factor- $\beta$ signaling in myogenic cells regulates vascular morphogenesis, differentiation, and matrix synthesis. Arterioscler Thromb Vasc Biol 32:e1-11.

Leone AM, Rutella S, Bonanno G, et al. (2006). Endogenous G-CSF and CD34+ cell mobilization after acute myocardial infarction. Int $\mathrm{J}$ Cardiol 111:202-8.

López B, González A, Varo N, et al. (2001). Biochemical assessment of myocardial fibrosis in hypertensive heart disease. Hypertension 38: 1222-6.

Luo DD, Phillips A, Fraser D. (2010). Bone morphogenetic protein-7 inhibits proximal tubular epithelial cell Smad3 signaling via increased SnoN expression. Am J Pathol 176:1139-47.

McLenachan JM, Dargie HJ. (1990). Ventricular arrhythmias in hypertensive left ventricular hypertrophy. Relationship to coronary artery disease, left ventricular dysfunction, and myocardial fibrosis. Am J Hypertens 3:735-40.

Moon JC, Mogensen J, Elliott PM, et al. (2005). Myocardial late gadolinium enhancement cardiovascular magnetic resonance in hypertrophic cardiomyopathy caused by mutations in troponin I. Heart 91:1036-40.
Moon JC, Sachdev B, Elkington AG, et al. (2003). Gadolinium enhanced cardiovascular magnetic resonance in Anderson-Fabry disease. Evidence for a disease specific abnormality of the myocardial interstitium. Eur Heart J 24:2151-5.

Moser M, Yu Q, Bode C, et al. (2007). BMPER is a conserved regulator of hematopoietic and vascular development in zebrafish. J Mol Cell Cardiol 43:243-53.

Myllärniemi M, Lindholm P, Ryynänen MJ, et al. (2008). Gremlinmediated decrease in bone morphogenetic protein signaling promotes pulmonary fibrosis. Am J Respir Crit Care Med 177:321-9.

Olivetti G, Melissari M, Balbi T, et al. (1994). Myocyte cellular hypertrophy is responsible for ventricular remodelling in the hypertrophied heart of middle aged individuals in the absence of cardiac failure. Cardiovasc Res 28:1199-208.

Onichtchouk D, Chen YG, Dosch R, et al. (1999). Silencing of TGF-beta signalling by the pseudoreceptor BAMBI. Nature 401:480-5.

Pegorier S, Campbell GA, Kay AB, Lloyd CM. (2010). Bone morphogenetic protein (BMP)-4 and BMP-7 regulate differentially transforming growth factor (TGF)-beta1 in normal human lung fibroblasts (NHLF). Respir Res 11:85-96.

Querejeta R, López B, González A, et al. (2004). Increased collagen type I synthesis in patients with heart failure of hypertensive origin: relation to myocardial fibrosis. Circulation 110:1263-8.

Rose RA, Jiang H, Wang X, et al. (2008). Bone marrow-derived mesenchymal stromal cells express cardiac-specific markers, retain the stromal phenotype, and do not become functional cardiomyocytes in vitro. Stem Cells 26:2884-92.

Rossi MA. (1998). Pathologic fibrosis and connective tissue matrix in left ventricular hypertrophy due to chronic arterial hypertension in humans. J Hypertens 16:1031-41.

Schwartzkopff B, Brehm M, Mundhenke M, Strauer BE. (2000). Repair of coronary arterioles after treatment with perindopril in hypertensive heart disease. Hypertension 36:220-5.

Sekiya T, Oda T, Matsuura K, Akiyama T. (2004). Transcriptional regulation of the TGF-beta pseudoreceptor BAMBI by TGF-beta signaling. Biochem Biophys Res Commun 320:680-4.

Tanaka M, Fujiwara H, Onodera T, et al. (1986). Quantitative analysis of myocardial fibrosis in normals, hypertensive hearts, and hypertrophic cardiomyopathy. Br Heart J 55:575-81.

Wang S, Sun A, Li L, et al. (2012). Up-regulation of BMP-2 antagonizes TGF- $\beta 1 /$ ROCK-enhanced cardiac fibrotic signalling through activation of Smurf1/Smad6 complex. J Cell Mol Med 16: 2301-10.

Wasserman K, Hansen JE, Sue DY, et al., eds. (2005). Principles of exercise testing and interpretation including pathophysiology and clinical applications. 4th ed. Philadelphia (PA): Lippincott Williams \&Wilkins.

Weber KT. (2000). Fibrosis and hypertensive heart disease. Curr Opin Cardiol 15:264-72.

Weidemann F, Breunig F, Beer M, et al. (2005). The variation of morphological and functional cardiac manifestation in Fabry disease: potential implications for the time course of the disease. Eur Heart $\mathbf{J}$ 26:1221-7.

Wilkinson L, Kolle G, Wen D, et al. (2003). CRIM1 regulates the rate of processing and delivery of bone morphogenetic proteins to the cell surface. J Biol Chem 278:34181-8.

Xavier S, Gilbert V, Rastaldi MP, et al. (2010). BAMBI is expressed in endothelial cells and is regulated by lysosomal/autolysosomal degradation. PLoS One 5:e12995.

Yanagisawa H, Davis EC. (2010). Unraveling the mechanism of elastic fiber assembly: the roles of short fibulins. Int J Biochem Cell Biol 42: 1084-93.

Yang T, Chen SL, Lu XJ, et al. (2012). Bone morphogenetic protein 7 suppresses the progression of hepatic fibrosis and regulates the expression of gremlin and transforming growth factor $\beta 1$. Mol Med Rep 6:246-52.

Yang YL, Ju HZ, Liu SF, et al. (2011). BMP-2 suppresses renal interstitial fibrosis by regulating epithelial-mesenchymal transition. J Cell Biochem 112:2558-65.

Zeisberg M, Hanai J, Sugimoto H, et al. (2003). BMP-7 counteracts TGF-beta1-induced epithelial-to-mesenchymal transition and reverses chronic renal injury. Nat Med 9:964-8.

Zhao T, Zhao W, Chen Y, et al. (2011). Acidic and basic fibroblast growth factors involved in cardiac angiogenesis following infarction. Int J Cardiol 152:307-13. 